\title{
A Routing Algorithm for Resource Constrained Wireless Self-organized Networks
}

\author{
https://doi.org/10.3991/ijoe.v13i11.7750 \\ Dan Han $\left.{ }^{(}\right)$, Lingmei Fu \\ Sanya Polytechnica Vacational College, Sanya, China \\ handan@ucsanya.com
}

\begin{abstract}
To explore the algorithms for wireless self-organized networks, through the NS-2 network simulation platform, the simulation analysis is made on the performances of multi hop data transmission of the existing four typical Ad hoc network routing protocols. And the energy saving strategy of Ad hoc network routing protocol SFR (Selection Forwarding Routing) based on network average node connection degree is discussed and analyzed, which is suitable for intensive Ad hoc network. The simulation data showed that, SFR protocol can, under the premise of ensuring the network coverage rate the same as that of flooding algorithm, reduce more than $56.1 \%$ redundant information in the network, and reduce the energy consumption of network by more than $30.2 \%$. Finally, it is concluded that the selection forwarding routing has quite good performance.
\end{abstract}

Keywords-NS-2 network simulation platform; Ad hoc network routing protocol; routing algorithm

\section{$1 \quad$ Introduction}

The energy saving strategy of routing protocols in Ad hoc network, in the NS-2 network simulation platform, the simulation analysis is made for on-demand routing protocols AODV and DSR, table driven routing protocol DSDV and temporary sequence routing protocol TORA based on the "height" concept. On the basis of simulation, the advantages and disadvantages of the four protocols and their applicable environment are summarized, and the detailed performance comparison is made. Especially in the energy consumption analysis, a completely new evaluation method Bec of energy consumption byte level that can accurately measure multi hop data transmission energy consumption is put forward. In addition, this method is used to make indepth analysis and comparison of energy consumption of four routing protocols. Based on the analysis of four typical Ad hoc network routing protocol and typical Flooding broadcast protocol, the routing protocol Selection Forwarding Routing is proposed based on the network average node network connectivity, which is suitable for intensive Ad hoc network. In this protocol, unlike the traditional routing protocols, it is no longer that all the nodes are forwarding routing look-up messages, but only a few nodes that meet the connectivity conditions continue to forward the routing look- 
up message. After the destination node received the routing look-up message, the destination node is not directly back to the link message along the original direction for the source node. Instead, it first of all launched a routing shortcut look-up processing, to get the closer shortcut routing with little amount of messages, and then the link message of routing shortcut is returned to the source node. Compared with the traditional Internet routing protocols, the main design goal of Ad hoc network routing protocol is to node mobility and maintain high performance in different mobile environment. As a result, in the simulation, we mainly study the impact of node mobility and network size on the performance of the routing protocol. This paper uses the simulation platform to study the energy saving performance of routing protocols in Ad hoc networks, and then analyzes the situations of reducing routing energy consumption.

\section{Literature review}

Singla and other scholars have simulated and compared the performance of table driven routing protocol DSDV and demand driven routing protocol AODV and TORA in different message traffic environments [2]. Morshed and his partners used the delay and shaking as the evaluation indexes to simulate the performance of DSDV and AODV. However, none of them evaluated the energy consumption of multi hop packet data transmission under protocol control. Pascoe-Chalke [4] put forward several models that can simulate the movement of nodes in Ad hoc networks, but they did not use these models to simulate the performance of routing protocols. Khan and others have simulated the performance of on-demand routing protocol AODV and table driven routing protocol OLSR in vehicular Ad hoc networks (VANETs). During the simulation, they introduced several mobility models to simulate the VANETs. Unlike this paper, the models they used are limited to simulations of VANETs, unable to really simulate the Ad hoc networks. The network scale model and the node mobility model are used to simulate the actual Ad hoc network, and the performance of the routing protocol is simulated and analyzed. Because the number of nodes in Ad hoc network is large, and the price of each node in the actual condition is more expensive, we use NS-2 network simulation platform to simulate the Ad hoc network.

\section{Method}

\subsection{Energy consumption evaluation method Bec for Wireless Ad Hoc Networks}

We defined a new index Bec(Energy Consume of OneByte) to evaluate the node energy consumption, shown as follows: 


$$
B_{e c}=\frac{\sum_{i=0}^{x}\left(E(A G T)_{i} * h o p s_{i}\right)+\sum_{j=0}^{y}\left(E(R T R)_{j} * \text { hops }_{j}\right)+\sum_{k=0}^{z}\left(E(M A C)_{k} * h o p s_{k}\right)}{\left(N^{*} \text { Size }\right)}
$$

In the above formula, $\mathrm{i}$ represents the $\mathrm{i}$-th business layer data packet transmitted at the service layer, and E(AGT) refers to the energy needed for each node to send the ith business layer data packet, whose value is related to the size of the data packet. hops is the number of hops required for the $\mathrm{i}$-th business layer data packet from the source node to the destination node. E(AGT) *hops indicates the total amount of energy consumed by the $\mathrm{i}$-th business layer data packet after a multi hop transmission from the source node to the destination node. Similarly, $j$ represents the $j$-th routing protocol packet transmitted at the routing layer and the MAC layer, and k suggests the $\mathrm{k}$-th MAC layer data packet transmitted at the MAC layer, respectively. E(RTR) and $\mathrm{E}(\mathrm{MAC})$ refer to the energy consumed for each node to send the $\mathrm{j}$-th routing layer protocol packet and the k-th MAC layer packet. E(RTR) *hops and E(MAC) k*hops represent the total energy consumed for the $\mathrm{j}$-th routing protocol packet and the $\mathrm{k}$-th MAC layer data transmitted in the MAC layer, after the multi-hop transmission, from the source node to the destination node. $\mathrm{x}$ and $\mathrm{y}$ indicate the number of packets of the business layer, routing layer, and MAC layer. The sum of energy consumption value of all the packets of business layer, routing layer, and MAC layer is the energy consumption value for the whole network to make message transmission.

\subsection{Static model}

In the static model, we set that the node is distributed according to the matrix form, the spacing between node is 150 meters, and the number of nodes were $6,12,20,30$, $42,56,72,90,100,121$, and 144 , respectively. The data stream is transmitted according to the diagonal direction. In the static model, we compare the performance of AODV and DSR two protocols mainly from the routing discovery time delay, the number of hops of routing link redundancy, and network energy consumption 3 aspects.

Route discovery time delay in static environment: the routing discovery timr delay of AODV is better than that of DSR in the case of different number of nodes. The performance of the two protocols is basically the same. In the case of fewer nodes, the optimal path can be selected, and the number of redundant hops increases with the number of nodes increasing. The number of redundant hops between the two protocols is no more than $33.34 \%$ of the optimal path. Energy consumption $(\mathrm{Be})$ in static environment: in stationary state, when the number of nodes is less, the energy consumption of AODV and DSR is basically the same, and when the number of nodes is more, the energy consumption of DSR increases rapidly. 


\subsection{Performance comparison of various kinds of Ad hoc routing protocol}

In order to analyze the applicability of Ad hoc routing protocol in different types in complex wireless networks environment, in this part, we establish two models of analog simulation of complex wireless Ad hoc network environment: the network scale model and node mobility model. In addition, the performance of Ad hoc routing protocol in different types is studied from the applicability of networks in different scales, the applicability of different node moving speeds, and the applicability of pause time of different nodes. This part, based on the network scale model and node mobility model, makes use of end-to-end delay, end-to-end delivery rate and network energy consumption three parameters. In addition, the applicability of AODV, DSR, DSDV and TORA four routing protocols in complex wireless ad hoc network environment is compared and analyzed in detail.

We will make the performance simulation and comparison of 4 protocols in 3 models according to the network scale model, the node moving speed model and the node static time model.

The network scale model: in the network scale model, in order to simulate all kinds of large-scale Ad hoc networks, the number of nodes is set to 16, 36, 64 and 100, and the network topology range is dynamically adjusted according to the number of nodes. The model with 16 nodes is to simulate the small scale wireless ad hoc network, the model with 36 and 64 nodes is designed to simulate the medium scale network, and the model with 100 nodes is used to simulate large networks. All nodes in the network move at the same fixed speed $(15 \mathrm{~m} / \mathrm{s})$, and the static time of all nodes is set to 0 . That is to say, all nodes are moving constantly.

The node mobile speed model: we consider the mobility problem of Ad hoc network respectively from two angles of node mobile speed and node static time. In the node mobile speed model, the applicability of the performance of routing protocols in different node mobile speed environment is analyzed. In this model, the network size is taken the medium size (49 nodes). The moving speed of nodes is $0 \mathrm{~m} / \mathrm{s}, 5 \mathrm{~m} / \mathrm{s}$, $20 \mathrm{~m} / \mathrm{s}, 45 \mathrm{~m} / \mathrm{s}$ and $80 \mathrm{~m} / \mathrm{s}$, respectively. When the node speed is $0 \mathrm{~m} / \mathrm{s}$, the network is a completely static environment, and the simulation parameters can show the performance of the routing protocol in the static wireless sensor network, and the performance of four routing protocols under node moving speed model.

The node static time model: the static feature of node refers to that any node randomly moves to a place and continues the random movement after being static for a period of time. The introduction of node static time model allows the simulation of the wireless ad hoc network more close to the actual situation, which increases the simulation accuracy. In this model, a total of 11 scenes are formed with every 1 second as intervals. In order to make the simulation more universal, we set the network size to 49 nodes, and the node moving speed is $1 \mathrm{~m} / \mathrm{s}$. Compared with the terminal time delay in the node static time model, the end-to-end delay of AODV is almost unaffected by the static time of nodes, and has always been maintained at a low time. But the end-to-end delay of other three protocols decreased in different degrees with node static time growth. The influence on DSDV is the most obvious. When the node static time is one second, the end-to-end delay of DSDV is still increased by 1.83 
times than that of completely static. In the static time model, the end-to-end delivery rate of the DSR protocol has been maintained at over $96 \%$, the AODV protocol has been maintained at over $93 \%$, and the TORA protocol has been maintained at over $90 \%$. The end-to-end delivery rate of DSR protocol in node time static model is slightly better than that of AODV and TORA. The change of node static time has little influence on the performance of the above 3 protocols, but it has a great impact on the DSDV protocol.

\section{$4 \quad$ Results and discussion}

\subsection{Performance changes of AODV under mobile model}

The number of nodes in the mobile model is set to $16,36,64$, and 100 , and each scene is simulated under four moving speeds $(5 \mathrm{~m} / \mathrm{s}, 20 \mathrm{~m} / \mathrm{s}, 45 \mathrm{~m} / \mathrm{s}$, and $80 \mathrm{~m} / \mathrm{s})$, a total of 16 mobile environments. In order to verify the relationship between protocol performance and node mobility, the dwell time of nodes is set to 0 seconds, that is, all nodes move at constant speed. In order to prevent the node distance too far for the communication, the range of network topology is set to a dynamic values, which is defined as follows: Simulation topo $=150 \sqrt{\mathrm{n} x} 150 \sqrt{\mathrm{n}}$ where $\mathrm{n}$ is the number of node. In this paper, we mainly focus on vertical comparison of three indicators, the AODV end-to-end delivery rate, end-to-end delay and energy consumption ec. The objective is to analyze the performance and difference of wireless ad hoc network under the control of AODV protocol in different mobile environment.

From the above analysis, it is known that, when the network scale is smaller, the impact of node moving speed on AODV's packet transmission performance and network energy consumption is little. While in the large scale network situation, the performance of protocol multi hop data transmission and the performance of energy consumption decrease with the increase of the node move speed.

\subsection{Performance change of DSR protocol under mobile model}

In this paper, the mobile model is totally the same as AODV. The vertical comparison of three indicators DSR protocol end-to-end delivery rate, end-to-end delay and energy consumption ec is made, so as to analyze the performance and difference of wireless ad hoc network under the control of DSR protocol in different mobile environment. The end-to-end delay of DSR protocol in the mobile model, when it moves at a rate of 0 , end-to-end delay of DSR increases slightly with the increase of number of nodes. In low speed conditions, the performance is basically stable, which is slightly affected by the number of nodes. At high speed, the delay increased 3 times larger than that at low speed. When the node number is 100 , and the moving speed is $80 \mathrm{~m} / \mathrm{s}$, the end-to-end delay is 3.44 times that when the speed is $20 \mathrm{~m} / \mathrm{s}$. The end-to-end delay of DSR is obviously affected by the moving speed, and the more the number of nodes, the greater the influence. Under the mobile model, the end-to-end delivery rate of DSR protocol is: when the number of nodes is less than 36 , the delivery rate remains 
above $94.3 \%$ regardless of the speed of movement. When the 100 node is at speed $45 \mathrm{~m} / \mathrm{s}$, the delivery rate drops to $39.9 \%$. The analysis shows that the more the number of nodes is, the greater the effect of speed on end-to-end delivery rate of DSR is. The simulation result shows that the multi hop data transmission and energy consumption performance of DSR protocol are slightly affected by moving speed when the network scale is small. While when the network scale is large, the protocol performance significantly decreased with the increase of node speed.

\subsection{SFR performance simulation}

Figure 1 shows that the total energy consumption of each hop network of the SFR protocol is obviously lower than that of flooding mode. When the routing discovery process is completed, the total energy consumption of flooding is 5633 , which is 1.43 times of that of SFR protocol. At the completion of the third hop, the total network energy consumption of flooding is 1.71 times that of SFR. The SFR protocol can save $30.2 \%$ of the total energy of the network, while ensuring the same coverage as flooding.

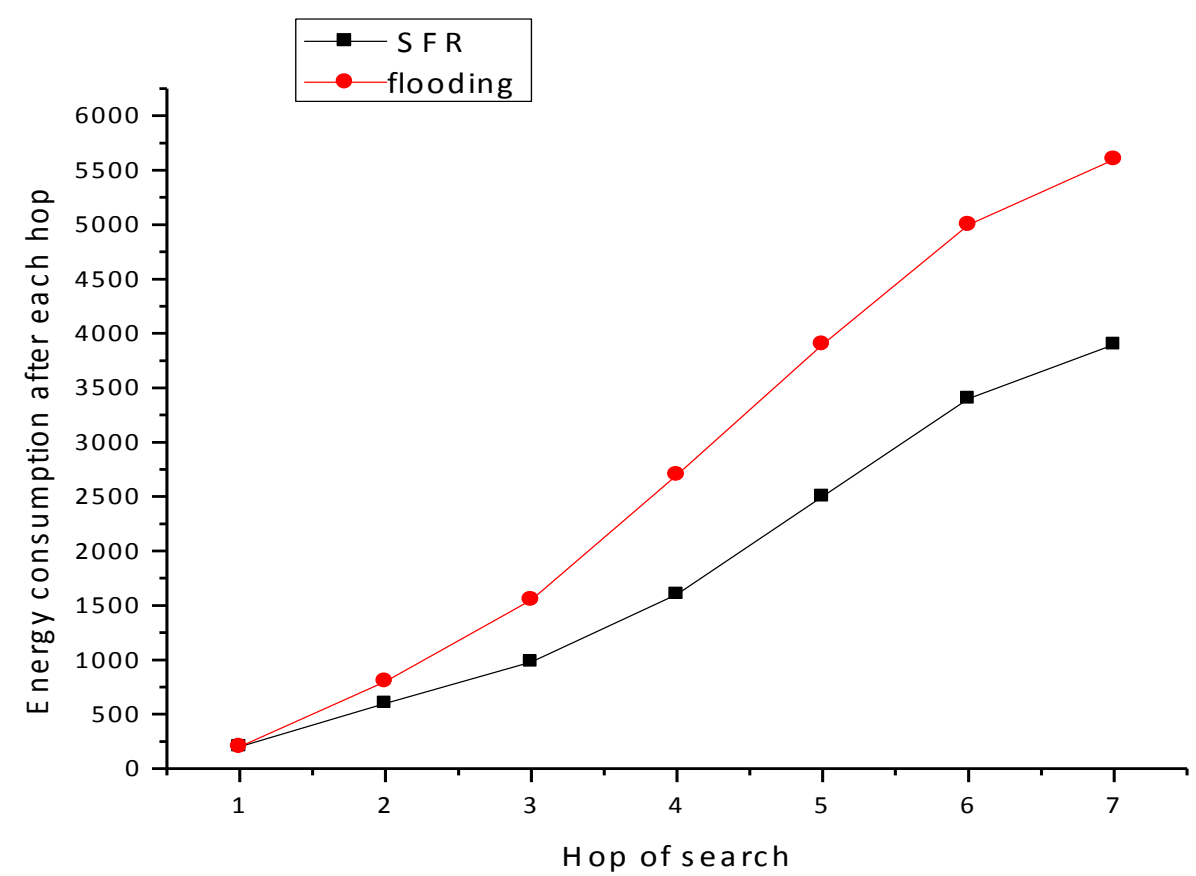

Fig. 1. The total energy consumption of each hop network of the SFR protocol 


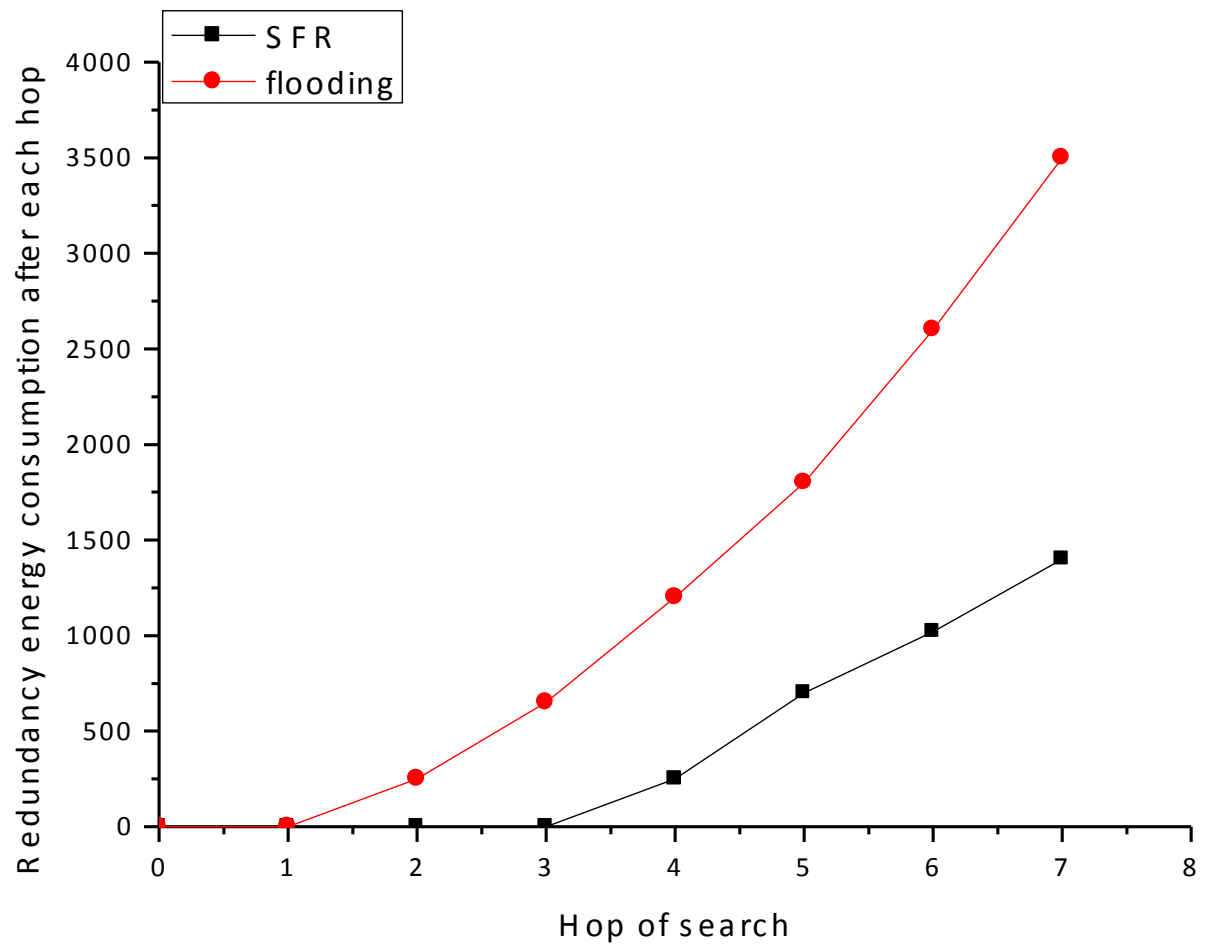

Fig. 2. The redundant energy consumption of each hop network of the SFR protocol

As shown in figure 2, it is the redundant energy consumption per jump network. It clearly shows that the amount of redundant energy consumed by flooding increases rapidly as the number of hops increases, especially between the third to sixth hops. The SFR protocol does not have any redundant messages in the first few hops, and only there are only a small amount of redundant messages at the beginning of the fourth hop. The total redundancy energy consumption of flooding is 2.27 times that of SFR routing protocol. Compared with flooding algorithm, SFR routing protocol can reduce more than $56.1 \%$ redundant messages.

\section{Conclusion}

In this paper, we mainly study the energy consumption control strategy of wireless ad hoc networks from the point of view of routing protocols. We propose an energy consumption controlled routing protocol SFR (Selection, Forwarding Routing) suitable for dense Ad hoc networks. In order to evaluate the performance of SFR protocol in detail, the performance simulation and analysis of SFR are made. Based on network simulator NS-2, 10 intensive Ad hoc network models with different network scales and different node connection degrees are constructed. And the performance of SFR is simulated and analyzed, and the Flooding is chosen as the performance to 
compare the network coverage after each hop; the total energy consumption after each hop; and the energy redundancy rate after each hop.

It is believed that a routing protocol using segmentation search strategy in the routing discovery phase can be proposed. According to the average node network connectivity, forwarding rules are made. The forwarding of routing request messages, at the first several hops, tends to node with high node connection degree, so as to rapidly expand the network coverage range. At the latter several hops, the forwarding of routing request messages tends to nodes with low node connection degree, so as to ensure that the network can cover the areas with sparse distribution of nodes.

\section{References}

[1] Kontik, M., \& Ergen, S. C. (2017). Distributed Medium Access Control Protocol for Successive Interference Cancellation-Based Wireless Ad Hoc Networks. IEEE Communications Letters, 21(2), 354-357. https://doi.org/10.1109/lcomm.2016.2619702.

[2] Varshney, P. K., Agrawal, G. S., \& Sharma, S. K. (2016). Relative Performance Analysis of Proactive Routing Protocols in Wireless Ad hoc Networks using Varying Node Density. Invertis Journal of Science \& Technology, 9(3), 161-169. https://doi.org/10.5958/2454762x.2016.00015.9.

[3] Varshney, P. K., Agrawal, G. S., \& Sharma, S. K. (2016). Energy Consumption Analysis of Wireless Ad hoc Networks Routing Protocols under Different Energy Models. INROADS-An International Journal of Jaipur National University, 5(1s), 250-253. https://doi.org/10.5958/2277-4912.2016.00048.5.

[4] Yadav, A. K., Das, S. K., \& Tripathi, S. (2017). EFMMRP: Design of efficient fuzzy based multi-constraint multicast routing protocol for wireless ad-hoc network. Computer Networks, 118, 15-23. https://doi.org/10.1016/j.comnet.2017.03.001.

[5] Prusty, A. R., Sethi, S., \& Nayak, A. K. (2016). A hybrid multi-hop mobility assisted heterogeneous energy efficient cluster routing protocol for Wireless Ad hoc Sensor Networks. Journal of High Speed Networks, 22(4), 265-280. https://doi.org/10.3233/jhs-160548.

[6] Biagioni, E., Giordano, S., Luo, X., Camp, T., \& Tian, T. (2016). Social and mobile solutions in ad hoc and sensor networking [Series Editorial]. IEEE Communications Magazine, 54(7), 100-101. https://doi.org/10.1109/mcom.2016.7509385.

\section{$7 \quad$ Authors}

Dan Han and Lingmei Fu are with Sanya Polytechnica Vacational College, Sanya, China.

Article submitted 24 September 2017. Published as resubmitted by the authors 20 October 2017. 